\title{
Structure formation in the DGP cosmological model
}

\author{
Kazuya Koyama, Roy Maartens \\ Institute of Cosmology $\&$ Gravitation, University of Portsmouth, Portsmouth PO1 2EG, UK
}

(Dated: February 5, 2008)

\begin{abstract}
The DGP brane-world model provides an alternative to the standard LCDM cosmology, in which the late universe accelerates due to a modification of gravity rather than vacuum energy. The cosmological constant $\Lambda$ in LCDM is replaced by a single parameter, the crossover scale $r_{c}$, in DGP. The Supernova redshift observations can be fitted by both models, with $\Lambda \sim H_{0}^{2}$ and $r_{c} \sim H_{0}^{-1}$. This degeneracy is broken by structure formation, which is suppressed in different ways in the two models. There is some confusion in the literature about how the standard linear growth factor is modified in DGP. While the luminosity distance can be computed purely from the modified 4-dimensional Friedman equation, the evolution of density perturbations requires an analysis of the 5-dimensional gravitational field. We show that if the 5-dimensional effects are inappropriately neglected, then the 4-dimensional Bianchi identities are violated and the computed growth factor is incorrect. By using the 5-dimensional equations, we derive the correct growth factor.
\end{abstract}

\section{INTRODUCTION}

The acceleration of the late-time universe, as implied by observations of Supernovae redshifts, cosmic microwave background anisotropies and the large-scale structure, poses one of the deepest theoretical problems facing cosmology. Within the framework of general relativity, the acceleration must originate from a dark energy field with effectively negative pressure, such as vacuum energy or a slow-rolling scalar field ("quintessence"). So far, none of the available models has a natural explanation. For example, in the simplest option of vacuum energy, leading to the "standard" LCDM model, the incredibly small,

$$
\rho_{\Lambda, \mathrm{obs}}=\frac{\Lambda}{8 \pi G} \sim H_{0}^{2} M_{P}^{2} \ll \rho_{\Lambda, \text { theory }},
$$

and incredibly fine-tuned,

$$
\Omega_{\Lambda} \sim \Omega_{m}
$$

value of the cosmological constant cannot be explained by current particle physics.

An alternative to dark energy plus general relativity is provided by models where the acceleration is due to modifications of gravity on very large scales, $r \gtrsim H_{0}^{-1}$. One of the simplest covariant models is based on the Dvali-Gabadadze-Porrati (DGP) brane-world model 1], in which gravity leaks off the 4-dimensional Minkowski brane into the 5-dimensional "bulk" Minkowski spacetime at large scales. At small scales, gravity is effectively bound to the brane and 4-dimensional Newtonian dynamics is recovered to a good approximation. The transition from 4 - to 5 -dimensional behaviour is governed by a crossover scale $r_{c}$; the weak-field gravitational potential behaves as

$$
\Psi \sim \begin{cases}r^{-1} & \text { for } r<r_{c} \\ r^{-2} & \text { for } r>r_{c}\end{cases}
$$

The DGP model was generalized by Deffayet to a Friedman-Robertson-Walker brane in a Minkowski bulk
2]; the gravity leakage at late times initiates acceleration - not due to any negative pressure field, but due to the weakening of gravity on the brane. The energy conservation equation remains the same as in general relativity, but the Friedman equation is modified:

$$
\begin{aligned}
& \dot{\rho}+3 H(\rho+p)=0, \\
& H^{2}-\frac{H}{r_{c}}=\frac{8 \pi G}{3} \rho .
\end{aligned}
$$

It is important to stress that the modification to the Friedman equation is derived from a covariant 5dimensional action and junction conditions across the brane 2].

The modified Friedman equation (5) shows that at late times in a CDM universe, with $\rho \propto a^{-3} \rightarrow 0$, we have

$$
H \rightarrow H_{\infty}=\frac{1}{r_{c}} .
$$

Since $H_{0}>H_{\infty}$, in order to achieve acceleration at late times, we require $r_{c} \gtrsim H_{0}^{-1}$, and this is confirmed by fitting SN observations [3].

Like the LCDM model, the DGP model is simple, with a single parameter $r_{c}$ to control the late-time acceleration. The DGP model does not provide a natural solution to the late-acceleration problem; similarly to the LCDM model, where $\Lambda$ must be fine-tuned, the DGP parameter $r_{c}$ must be fine-tuned to match observation. Furthermore, it has been recognized that the DGP model suffers from potentially serious theoretical problems, such as the existence of a ghost in de Sitter solutions of Eq. (5) and the strong coupling problem [4]. Nevertheless, like LCDM, the DGP is a simple covariant model that makes testable predictions, in particular the prediction of latetime acceleration. (It is worth noting that the original DGP model with a Minkowski brane was not introduced to explain acceleration - the generalization to a Friedman brane was subsequently found to be self-accelerating.)

LCDM and DGP can both account for the SN observations, with $\Lambda \sim H_{0}^{2}$ and $r_{c} \sim H_{0}^{-1}$ respectively. This de- 
generacy may be broken by observations based on structure formation, since the two models predict different suppression of density perturbations. For LCDM, the analysis of density perturbations is well understood. For DGP it is much more subtle and complicated; although matter is confined to the 4-dimensional brane, gravity is fundamentally 5-dimensional, and the bulk gravitational field responds to and backreacts on density perturbations. The background cosmological dynamics, and hence the predicted SN redshifts, can be treated purely via the modified 4-dimensional Friedman equation. By contrast, the evolution of density perturbations requires an analysis based on the 5-dimensional nature of gravity.

\section{PERTURBATION EQUATIONS ON THE BRANE}

Effective covariant equations on the brane, for an arbitrary brane metric and matter distribution, can be derived by projecting the 5 -dimensional Einstein equations $\left(G_{a b}^{(5)}=0\right)$ and applying the Israel junction conditions with reflection symmetry at the brane [5, 6]

$$
G_{\mu \nu}=\left(16 \pi G r_{c}\right)^{2} \Pi_{\mu \nu}-E_{\mu \nu}
$$

where

$$
\begin{aligned}
\tilde{T}_{\mu \nu}= & T_{\mu \nu}-(8 \pi G)^{-1} G_{\mu \nu} \\
\Pi_{\mu \nu}= & -\frac{1}{4} \tilde{T}_{\mu \alpha} \tilde{T}_{\nu}^{\alpha}+\frac{1}{12} \tilde{T}_{\alpha}^{\alpha} \tilde{T}_{\mu \nu} \\
& +\frac{1}{24}\left[3 \tilde{T}_{\alpha \beta} \tilde{T}^{\alpha \beta}-\left(\tilde{T}_{\alpha}^{\alpha}\right)^{2}\right] g_{\mu \nu},
\end{aligned}
$$

and $E_{\mu \nu}$ is the trace-free projection of the 5D Weyl tensor. The energy-momentum tensor satisfies

$$
\nabla^{\mu} T_{\mu \nu}=0
$$

as in general relativity. The 4D Bianchi identity imposes the constraint

$$
\nabla^{\mu} E_{\mu \nu}=\left(16 \pi G r_{c}\right)^{2} \nabla^{\mu} \Pi_{\mu \nu}
$$

In the background spacetime, $E_{\mu \nu}=0$ and Eq. (7) implies the modified Friedman equation (5). The perturbed FRW brane has a nonzero $E_{\mu \nu}$, which encodes the effects on the brane of the bulk gravitational field. The perturbed 5D field equations are needed to determine the evolution of $\delta E_{\mu \nu}$. We can parametrize the (gauge-invariant) scalar perturbations of $E_{\mu \nu}$ as an effective fluid, with density perturbation $\delta \rho_{E}$, isotropic pressure perturbation $\frac{1}{3} \delta \rho_{E}$, anisotropic stress perturbation $\delta \pi_{E}$ and energy flux perturbation $\delta q_{E}$ (see the appendix). Scalar metric perturbations are given in longitudinal gauge by

$$
d s^{2}=-(1+2 \Psi) d t^{2}+a^{2}(1+2 \Phi) d \vec{x}^{2},
$$

and the perturbed energy-momentum tensor for matter is given by

$$
\delta T_{\nu}^{\mu}=\left(\begin{array}{cc}
-\delta \rho & a \delta q_{, i} \\
-a^{-1} \delta q^{, i} & \delta p \delta_{j}^{i}
\end{array}\right)
$$

The Bianchi identity (11) gives evolution equations A2 and A3 for $\delta \rho_{E}$ and $\delta q_{E}$. The perturbed $4 \mathrm{D}$ field equations (7) imply a modified Poisson equation (A4) and constraint equation (16). The 4D perturbation equations are not closed, due to the presence of Weyl anisotropic stress $\delta \pi_{E}$. The 5D perturbation equations are needed to determine this stress. This is an extremely complicated problem in full generality, and especially on large scales where the 5D effects become strong. For the structure formation problem, we focus on subhorizon scales, with $k / a \ll H^{-1}$ and $k / a \ll r_{c}$, where $k$ is the comoving wavenumber. In addition, we use the quasi-static approximation which is relevant for structure formation and neglect time-derivatives terms relative to gradient terms. Then Eq. A2 implies

$$
\delta q_{E}=0 .
$$

With $\delta q_{E}=0$, the key perturbation equations reduce to

$$
\begin{aligned}
& \frac{k^{2}}{a^{2}} \Phi=4 \pi G\left(\frac{2 H r_{c}}{2 H r_{c}-1}\right)\left(\rho \triangle-\frac{\delta \rho_{E}}{2 H r_{c}}\right), \\
& \Phi+\Psi=8 \pi G \frac{H}{r_{c}\left(\dot{H}+2 H^{2}\right)-H} a^{2} \delta \pi_{E}, \\
& k^{2} \delta \pi_{E}-\frac{1}{2} \delta \rho_{E}=r_{c} \frac{\dot{H}}{H} F, \\
& \ddot{\triangle}+2 H \triangle=-\frac{k^{2}}{a^{2}} \Psi,
\end{aligned}
$$

where

$$
F \equiv \frac{\rho \triangle}{1-2 H r_{c}}+\frac{H}{r_{c}\left(2 H^{2}+\dot{H}\right)-H} k^{2} \delta \pi_{E}+\frac{\delta \rho_{E}}{2 H r_{c}-1}
$$

and the (gauge-invariant) comoving density perturbation is

$$
\rho \Delta=\delta \rho-3 H a \delta q .
$$

The equations (15)-(18) are not closed since $\delta E_{\mu \nu}$ is not determined by purely $4 \mathrm{D}$ equations. The ad hoc assumption $\delta E_{\mu \nu}=0$, i.e.,

$$
\delta \rho_{E}=\delta \pi_{E}=0,
$$

has been effectively adopted in some papers [7]. However, it is manifest that this assumption is not consistent with the Bianchi identity Eq. (17). Thus the analysis based on this assumption must be revisited.

Within our approximations, the Bianchi identity suggests that there exists a family of solutions characterized by

$$
\delta \rho_{E}=C\left(H r_{c}\right) k^{2} \delta \pi_{E},
$$


where $C\left(H r_{c}\right)$ is some function of $H r_{c}$. The correct $\mathrm{C}\left(H r_{c}\right)$ can be obtained only when we properly consider the $5 \mathrm{D}$ equations of motion.

\section{THE 5-DIMENSIONAL EQUATIONS}

The 5D background Minkowski metric is given by

$$
d s_{(5)}^{2}=-n(y, t)^{2} d t^{2}+b(y, t)^{2} d \vec{x}^{2}+d y^{2},
$$

where the brane is at $y=0$ and

$$
b=a(1+H y), \quad n=1+\left(\frac{\dot{H}}{H}+H\right) y .
$$

Because the background bulk is just a Minkowski spacetime, we can easily find a Mukohyama master variable $\Omega(t, \vec{x}, y)$ [] , from which all the solutions for the metric perturbations can be constructed (see the appendix). In the quasi-static approximation relevant for structure formation, we can neglect $\dot{\Omega}$ relative to $\Omega^{\prime}$, and the $5 \mathrm{D}$ wave equation for $\Omega$ becomes

$$
\Omega^{\prime \prime}+\left(-3 \frac{b^{\prime}}{b}+\frac{n^{\prime}}{n}\right) \Omega^{\prime}-\frac{k^{2}}{b^{2}} \Omega=0
$$

where a dot denotes a derivative with respect to time $t$ and a prime a derivative with respect to $y$. In the quasistatic approximation, the solution is

$$
\Omega=c_{1}\left[(1+H y)^{-k / a H}+c_{2}(1+H y)^{k / a H}\right] .
$$

Regularity of the bulk perturbations as $y \rightarrow \infty$ requires that $c_{2}=0$.

Perturbations of the Weyl fluid $\delta E_{\mu \nu}$ can be expressed in terms of $\Omega$,

$$
\begin{aligned}
& 8 \pi G \delta \rho_{E}=\left.\frac{k^{4}}{3 a^{5}} \Omega\right|_{y=0}, \\
& 8 \pi G \delta \pi_{E}=\frac{1}{6 a^{3}}\left(\frac{k^{2}}{a^{2}} \Omega-\frac{3 \dot{H}}{H} \Omega^{\prime}\right)_{y=0} .
\end{aligned}
$$

By Eq. A14 with $c_{2}=0$, we can neglect the last term on the right of Eq. (28). It follows that

$$
\delta \rho_{E}=2 k^{2} \delta \pi_{E},
$$

and we have determined the function $C$ in Eq. (22) as $C=2$.

\section{THE GROWTH FACTOR}

In the appendix, we find the solution for $\Omega$ (A15) by imposing the perturbed junction condition A13). Then using Eqs. (A7), (A8), A11, A12 and (A14, the solutions for the brane metric perturbations are

$$
\begin{aligned}
& \frac{k^{2}}{a^{2}} \Phi=4 \pi G\left(1-\frac{1}{3 \beta}\right) \rho \triangle \\
& \frac{k^{2}}{a^{2}} \Psi=-4 \pi G\left(1+\frac{1}{3 \beta}\right) \rho \triangle
\end{aligned}
$$

where

$$
\beta=1-2 r_{c} H\left(1+\frac{\dot{H}}{3 H^{2}}\right) .
$$

This agrees with the results obtained by Lue, Scoccimarro and Starkman [9]. They find spherical symmetric solutions by closing the $4 \mathrm{D}$ equations using an anzatz for the metric and checked in retrospect that the obtained solutions satisfy the regularity in the bulk. Here we have shown that the solutions (30) and (31) are uniquely determined by the regularity condition in the bulk within our approximations.

We can check that our solutions are consistent with the Bianchi identity. The Bianchi identity Eq. (17) gives nontrivial equations for $\delta \rho_{E}$ and $\delta \pi_{E}$ with the condition (29) and they can be solved in terms of $\rho \triangle$. Then substituting the solutions into Eqs. (15) and (16), we arrive at the solutions for $\Phi$ and $\Psi$ as in Eqs. (30) and (31).

The modified Poisson equation (30) shows the suppression of growth due to gravity leakage. The rate of growth is determined by $\Delta$, and for CDM,

$$
\ddot{\triangle}+2 H \dot{\triangle}=-\frac{k^{2}}{a^{2}} \Psi \text {. }
$$

which leads to

$$
\ddot{\triangle}+2 H \dot{\triangle}=4 \pi G\left(1+\frac{1}{3 \beta}\right) \rho \triangle .
$$

Thus the growth rate receives an additional modification from the time variation of Newton's constant through $\beta$. This effect can be described by a linearized scalar-tensor gravity with Brans-Dicke parameter [9]

$$
\omega=\frac{3}{2}(\beta-1),
$$

where the gravitational scalar corresponds to the bending of the brane Eq. A9.

In Fig. 1, we show the linear growth factor $\Delta / a$ for the DGP model, and compare it with LCDM, with the incorrect DGP result (in which the inconsistent assumption $\delta E_{\mu \nu}=0$ is effectively adopted), and with the general relativity dark energy model whose background evolution matches that of the DGP model (see Fig. 2).

\section{CONCLUSION}

We have shown by a careful analysis of the $5 \mathrm{D}$ perturbation equations that subhorizon density perturbations in the DGP cosmological models evolve according 


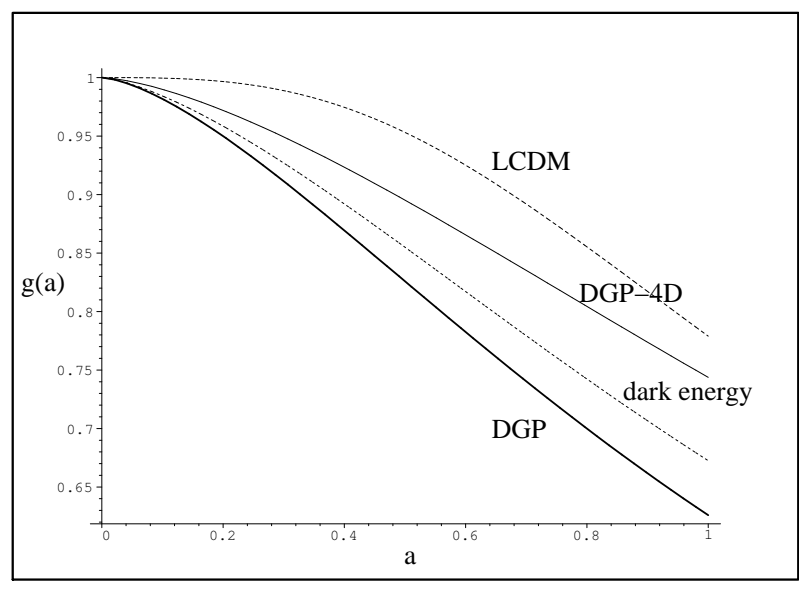

FIG. 1: The growth history $g(a)=\triangle(a) / a$ is shown for LCDM (long dashed) and DGP (solid, thick). The growth history for a dark energy model (short dashed) with the identical expansion histories with DGP is also shown (see Fig. 2) [10]. Due to the time variation of Newton's constant through $\beta$ in Eq. (34), the growth factor $g(a)$ receives an additional suppression compared with the dark energy model. DGP-4D (solid, thin) shows the incorrect result in which the inconsistent assumption $\delta E_{\mu \nu}=0$ is adopted. We set the desity parameter for matter today as $\Omega_{m 0}=0.3$.

to Eq. (34), that the modified Poisson equation is given by Eq. (30), and that the gravitational potential is given by Eq. (31). These expressions obey the constraint imposed by the $4 \mathrm{D}$ Bianchi identity, and they correct those expressions in the literature [7] which violate the Bianchi identity. Our results confirm the results derived via another approach by Lue, Scoccimarro and Starkman [9]. Although they also used the 5D equations to check consistency of their ansatz, they were not able to show, as we have done here, that their results follow uniquely from the $5 \mathrm{D}$ equations.

The correct equations for subhorizon density perturbations are crucial for meaningful tests of DGP predictions against structure formation observations. And such tests are essential for breaking the degeneracy with LCDM that arises with SN redshift observations [7, 10]. The distance-based SN observations draw only upon the background 4D Friedman equation (5) in DGP models, and therefore there are quintessence models in general relativity that can produce precisely the same SN redshifts as DGP (see Fig. 2). By contrast, structure formation observations require the $5 \mathrm{D}$ perturbations in DGP, and one cannot find equivalent general relativity models. Previous analyses of structure formation that are based on the incorrect equations for density perturbations [7] will need to be re-visited.

The linear growth factor, as shown in Fig.1, is the basis for tests of DGP against structure formation observations. But one also needs the metric perturbations. It

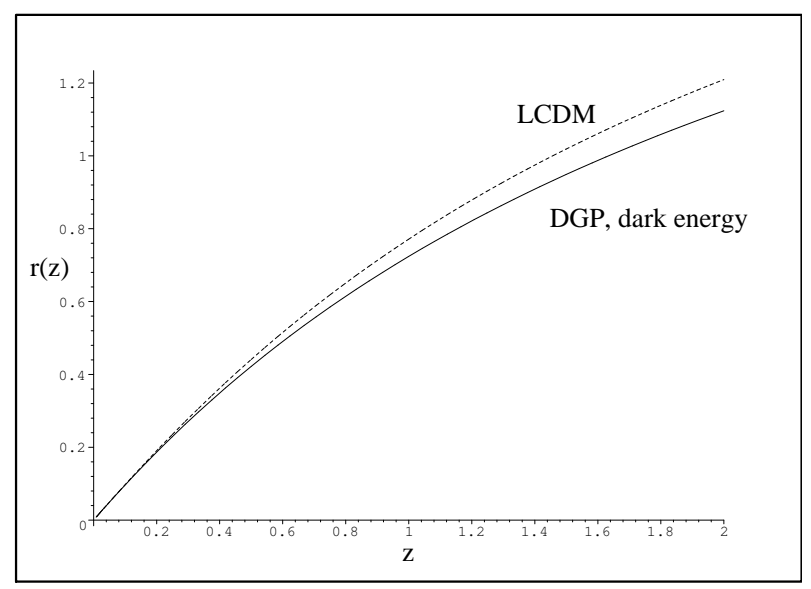

FIG. 2: The expansion history $r(z)=\int_{0}^{z} d z H(z)^{-1}$ is shown for LCDM (dashed) and DGP (solid) where $1+z=a^{-1}$. Expansion history for DGP can be mimicked by a dark energy model with the equation of state $w(a)=w_{0}+w_{a}(1-a)$ with $w_{0}=-0.78$ and $w_{a}=0.32[10]$.

follows from Eqs. (30) and (31) that

$$
\begin{aligned}
& \frac{k^{2}}{a^{2}}(\Phi-\Psi)=8 \pi G \rho \triangle \\
& \frac{k^{2}}{a^{2}}(\Phi+\Psi)=-\frac{8 \pi G}{3 \beta} \rho \triangle \propto \delta \pi_{E}
\end{aligned}
$$

where the proportionality follows from Eq. (16). Equation (37) differs from general relativity, where the absence of matter anisotropic stress implies $\Psi=-\Phi$. In the DGP model, the 5D gravitational field produces an anisotropic stress on the 4D universe. Equation (36) is the same as in general relativity - and this equation basically determines the integrated Sachs-Wolfe and weak lensing effects. This means that the formulas will take the same form as in general relativity, but the outcome will be different, because $\Delta$ will differ from the general relativity case.

We have also emphasized that our results (and those of Ref. 9]) apply on subhorizon scales. On superhorizon scales, where the 5D effects are strongest, the problem has yet to be solved. Thus a computation of CMB anisotropies will not be reliable on the largest scales.

Furthermore, we have restricted our discussion to the linear regime. In the nonlinear regime, the DGP theory approaches general relativity 11]. A full computation of weak lensing will require a careful matching of the linear DGP to the nonlinear general relativity regimes.

Acknowledgements: KK and RM are supported by PPARC. We thank Arthur Lue for useful discussions. 


\section{APPENDIX A: PERTURBATION EQUATIONS}

We can parametrize the scalar perturbations of $E_{\mu \nu}$ as an effective fluid,

$$
\delta E^{\mu}{ }_{\nu}=-8 \pi G\left(\begin{array}{cc}
-\delta \rho_{E} & a \delta q_{E, i} \\
a^{-1} \delta q_{E}{ }^{i} & \frac{1}{3} \delta \rho_{E} \delta^{i}{ }_{j}+\delta \pi_{E j}^{i}
\end{array}\right),
$$

where $\delta \pi_{i j}^{E}=\delta \pi_{, i j}^{E}-\frac{1}{3} \delta \pi_{E, k}^{, k} \delta_{i j}$. The $4 \mathrm{D}$ Bianchi identity Eq. (11) imposes

$$
\begin{aligned}
& \dot{\delta} \rho_{E}+4 H \delta \rho_{E}-a^{-1} k^{2} \delta q_{E}=0 \\
& \dot{\delta} q_{E}+4 H \delta q_{E}+a^{-1}\left(\frac{1}{3} \delta \rho_{E}-\frac{2}{3} k^{2} \delta \pi_{E}\right) \\
& =-a^{-1} \frac{2}{3} r_{c} \frac{\dot{H}}{H}\left\{-\frac{\rho \triangle}{2 H r_{c}-1}+\frac{\delta \rho_{E}}{2 H r_{c}-1}\right. \\
& \left.+\frac{1}{r_{c}(2 H+\dot{H} / H)-1} k^{2} \delta \pi_{E}\right\} .
\end{aligned}
$$

Combining the $(t, t)$ component and the $(0, i)$ component of the perturbed $4 \mathrm{D}$ field equations Eq. (7), we get the modified Poisson equation

$$
\frac{k^{2}}{a^{2}} \Phi=4 \pi G\left(\frac{2 H r_{c}}{2 H r_{c}-1}\right)\left(\rho \triangle-\frac{\delta \rho_{E}-3 H a \delta q_{E}}{2 H r_{c}}\right),
$$

where $\rho \triangle=\delta \rho-3 H a \delta q$. The traceless part of $(i, j)$ component gives Eq. (16). (We follow the standard assumption that the CDM anisotropic stress vanishes $\delta \pi=0$.)

In order to solve the perturbations in this background, it is convenient to use 5D Longitudinal gauge, given by

$$
\begin{aligned}
d s^{2}= & \left(1+2 A_{y y}\right) d y^{2}+n A_{y} d y d t-n^{2}\left(1+2 A^{2}\right) d t^{2} \\
& +b^{2}(1+2 \mathcal{R}) d \vec{x}^{2} .
\end{aligned}
$$

In Minkowski spacetime, if the metric perturbations are derived from a "master variable", $\Omega$, the perturbed $5 \mathrm{D}$ Einstein equations yield a single wave equation governing the evolution of the master variable $\Omega$ in the bulk:

$$
-\left(\frac{1}{n b^{3}} \dot{\Omega}\right)^{\cdot}+\left(\frac{n}{b^{3}} \Omega^{\prime}\right)^{\prime}-\frac{k^{2} n}{b^{5}} \Omega=0 .
$$

Within our approximation, we can neglect timederivative terms and the metric perturbations are written in terms of $\Omega$ as

$$
\begin{aligned}
& A=-\frac{1}{6 b}\left\{-3\left(\frac{n^{\prime}}{n}-2 \frac{b^{\prime}}{b}\right) \Omega^{\prime}+\frac{2 k^{2}}{b^{2}} \Omega\right\}, \\
& R=\frac{1}{6 b}\left(3 \frac{b^{\prime}}{b} \Omega^{\prime}+\frac{k^{2}}{b^{2}} \Omega\right) .
\end{aligned}
$$

An important feature of the DGP model is the branebending mode; in 5D longitudinal gauge, the location of the brane is perturbed and given by 12 ]

$$
y=\xi=-r_{c}(\Phi+\Psi) .
$$

Then the induced metric perturbations on the brane are given by

$$
\Psi=A-\left(\frac{\dot{H}}{H}+H\right) \xi, \quad \Phi=R-H \xi,
$$

from a gauge transformation. We can express $\Psi$ and $\Phi$ in terms of $A$ and $R$ as

$$
\begin{aligned}
& \Phi=\frac{1}{1-r_{c}\left(\frac{\dot{H}}{H}+2 H\right)}\left\{\left(1-r_{c} \frac{\dot{H}+H^{2}}{H}\right) R+H r_{c} A\right\}, \\
& \Psi=\frac{1}{1-r_{c}\left(\frac{\dot{H}}{H}+2 H\right)}\left\{\left(1-H r_{c}\right) A+r_{c} \frac{\dot{H}+H^{2}}{H} R\right\} .
\end{aligned}
$$

The perturbed junction condition is

$$
4 \pi G \rho \triangle=\frac{k^{2}}{a^{2}} \Phi+\frac{k^{2}}{2 a^{2}} \frac{\xi}{r_{c}}-\left.\frac{1}{4 r_{c}} \frac{k^{2}}{a^{3}} \Omega^{\prime}\right|_{y=0} .
$$

This junction condition gives the modified Poisson equation where the last two terms describe corrections from the $5 \mathrm{D}$ gravity.

Note that $\Omega^{\prime}$ appears in the expressions for $\delta \rho_{E}$ and $\delta \pi_{E}$. Unless we solve the $5 \mathrm{D}$ equations and impose proper boundary conditions, $\Omega$ and $\Omega^{\prime}$ on the brane are independent. This means that we can impose arbitrary conditions for the relation between $\delta \rho_{E}$ and $\delta \pi_{E}$. Indeed by choosing appropriate $c_{2}$ in Eq. (26), we can have any $C\left(H r_{c}\right)$ in Eq. 221). However, as described in the main text, the regularity condition impose $c_{2}=0$. Then we get

$$
H \Omega^{\prime} \ll \frac{k^{2}}{a^{2}} \Omega
$$

for $k / a H \gg 1$ and we can neglect the terms proportional to $\Omega^{\prime}$. However, the Poisson equation Eq. A13 still receives a correction from the brane bending mode $\xi$ and we get a linearized scalar-tensor gravity [13]. The junction condition Eq.

$$
\frac{k^{4} \Omega}{2 a^{5}}=\frac{1-2 H r_{c}\left(1+\dot{H} / 2 H^{2}\right)}{1-2 H r_{c}\left(1+\dot{H} / 3 H^{2}\right)} \kappa_{4}^{2} \rho \triangle .
$$

Then from Eqs. (A7), (A8), (A11) and A12, the solutions for metric perturbations can be obtained as Eqs. (30) and (31). We can also derive the solutions for $\delta \rho_{E}$ and $\delta \pi_{E}$ from Eqs. (27) and (28) and show that they are consistent with the $4 \mathrm{D}$ Bianchi identity. 
[1] G. R. Dvali, G. Gabadadze and M. Porrati, Phys. Lett. B 484, 112 (2000) arXiv:hep-th/0002190.

[2] C. Deffayet, Phys. Lett. B 502, 199 (2001) arXiv:hep-th/0010186.

[3] C. Deffayet, S. J. Landau, J. Raux, M. Zaldarriaga and P. Astier, Phys. Rev. D 66, 024019 (2002) arXiv:astro-ph/0201164.

[4] V. A. Rubakov, arXiv:hep-th/0303125

M. A. Luty, M. Porrati and R. Rattazzi, JHEP 0309 (2003) 029 arXiv:hep-th/0303116;

A. Nicolis and R. Rattazzi, JHEP 0406 (2004) 059 arXiv:hep-th/0404159;

K. Koyama, arXiv:hep-th/0503191

[5] T. Shiromizu, K. i. Maeda and M. Sasaki, Phys. Rev. D 62, 024012 (2000) arXiv:gr-qc/9910076.

[6] K. i. Maeda, S. Mizuno and T. Torii, Phys. Rev. D 68, 024033 (2003) arXiv:gr-qc/0303039.

[7] Y. S. Song, Phys. Rev. D 71, 024026 (2005) arXiv:astro-ph/0407489;

L. Knox, Y. S. Song and J. A. Tyson, arXiv:astro-ph/0503644

M. Ishak, A. Upadhye and D. N. Spergel, arXiv:astro-ph/0507184

I. Sawicki and S. M. Carroll, arXiv:astro-ph/0510364
[8] S. Mukohyama, Phys. Rev. D 62, 084015 (2000) arXiv:hep-th/0004067.

[9] A. Lue, R. Scoccimarro and G. D. Starkman, Phys. Rev. D 69, 124015 (2004) arXiv:astro-ph/0401515.

See also A. Lue, arXiv:astro-ph/0510068

A. Lue and G. Starkman, Phys. Rev. D 67, 064002 (2003) arXiv:astro-ph/0212083.

[10] E. V. Linder, Phys. Rev. D 72, 043529 (2005) arXiv:astro-ph/0507263.

[11] C. Deffayet, G. R. Dvali, G. Gabadadze and A. I. Vainshtein, Phys. Rev. D 65 (2002) 044026 arXiv:hep-th/0106001;

A. Lue, Phys. Rev. $\quad$ D $\quad 66 \quad$ (2002) 043509 arXiv:hep-th/0111168.

A. Gruzinov, New Astron. $10 \quad$ (2005) 311 arXiv:astro-ph/0112246;

T. Tanaka, Phys. Rev. D $69 \quad$ (2004) 024001 arXiv:gr-qc/0305031.

[12] C. Deffayet, Phys. Rev. D 66, 103504 (2002) arXiv:hep-th/0205084.

[13] C. Deffayet, Phys. Rev. D $71 \quad$ (2005) 103501 arXiv:gr-qc/0412114. 